\title{
Possibility of using mRNA expression levels for nucleic acid-metabolizing enzymes within prostate cancer cells as indices for prognostic factors
}

\author{
AKIRA YANO, YOSHINORI SHIGEMATSU, HIROYUKI KITANO, AKI HANAYAMA, \\ AKIRA OZAWA, TAKATOSHI TACHO and MOTOHIRO FUJII
}

Department of Urology, Matsuyama Red Cross Hospital, Ehime 790-8524, Japan

Received May 19, 2010; Accepted August 24, 2010

DOI: $10.3892 / \mathrm{ol} .2010 .165$

\begin{abstract}
Thymidylate synthase (TS), dihydropyrimidine dehydrogenase (DPD), thymidine phosphorylase (TP) and orotate phosphoribosyl transferase (OPRT) are enzymes involved in nucleic acid metabolism. It has been reported (based on observations of various tumor types) that the extent of the mRNA expression of these enzymes within tumor tissues may be used as a factor to define tumor prognosis. It has also been reported that the mRNA expression patterns differ in each type of tumor. However, few reports are available on the distribution of mRNA expression in prostate cancers. This study was conducted on tissue specimens obtained from 172 patients who were diagnosed with prostate cancer and had undergone total prostatectomies. The mRNA expression of TS, DPD, OPRT and TP was quantitatively analyzed using the Danenberg tumor profile (DTP) method. The results were used to examine the correlations between the distributions of the mRNAs and clinicopathological factors, as well as the significance of their expression as a prognostic factor. Patients with poorly differentiated cancers in their tissues showed a significant increase in the mRNA expression of TS and OPRT. The increases in the TP mRNA content were proportional to an increase in the Gleason scores. The prognosis was significantly poorer in those cases with a high expression of TS or OPRT mRNA and a low expression of DPD mRNA. In conclusion, the expression levels of mRNAs for TS, DPD and OPRT among the enzymes related to nucleic acid metabolism are useful as prognostic factors in patients with prostate cancers.
\end{abstract}

Correspondence to: Dr Akira Yano, Department of Urology, Matsuyama Red Cross Hospital, 1 Bunkyoumachi, Matsuyama, Ehime 790-8524, Japan

E-mail: redcross@dune.ocn.ne.jp

Key words: thymidylate synthase, dihydropyrimidine dehydrogenase, thymidine phosphorylase, orotate phosphoribosyl transferase, prostate cancer, prognostic factor

\section{Introduction}

Studies have been conducted on various factors involved in defining the prognosis or manipulating the effects of chemotherapy in order to improve the selection of therapeutic approaches and their outcomes in various types of tumors (1). The prognostic factors commonly assessed are clinicopathological, including tumor diameter, status of lymphatic metastasis and nuclear atypia. In cases of prostate cancer, tumor stage, the prognostic factors include Gleason scores and nuclear grades. For breast cancer, therapeutic modalities are selected by employing the factors that predict the efficacy of treatment. Such factors include immunohistochemical analysis of the expression of hormone receptors (ER and $\mathrm{PgR}$ ) that define the indication for endocrine therapy and testing for excessive expression of HER2 protein to determine the indication for the use of trastuzumab, a molecular-targeted therapeutic agent. For prostate cancers, however, no useful molecular markers, such as those cited above, exist to predict the outcome and efficacy of the drug therapy. DNA/RNA synthesis is essential for the proliferation of cancer cells, and for the enzymes that control the synthetic process, thymidylate synthase (TS), orotate phosphoribosyl transferase (OPRT) and thymidine phosphorylase (TP) in the de novo pathway are cited. Dihydropyrimidine dehydrogenase (DPD) has been identified for the enzymes involved in the breakdown of these nucleic acids. These enzymes play key roles in the metabolism of pyrimidine base, a component of DNA/RNA, while they are equally important in the metabolism of 5-FU anticancer agents (2-8).

In cancers involving the stomach, colon and breast, where 5-FU anticancer agents are considered to be the key drugs, the levels of expression of enzymes involved in nucleic acid metabolism, such as TS, DPD, TP and OPRT, have been investigated as markers for determining prognosis. Subsequently, numerous studies have been conducted on possible prognosis-deciding factors, as well as factors that predict the efficacy of 5-FU anticancer agents (13). On the other hand, it has been reported that the levels of expression of mRNA for these enzymes differ in each tumor (9), indicating a need to evaluate each tumor individually. 
Concerning prostate cancers, the expression levels of these enzymes may be related to prognosis. The expression of TS was evaluated in prostate cancers using a staining method to observe its relationship with the prognosis (10). This study suggested a potential role of TS as a prognostic factor.

The level of gene expression within tumor tissues may be elucidated when the mRNA of the target gene is extracted and its expression quantified using the real-time (RT)-PCR method. However, in prostate cancers, the prostate is characteristically dotted by tumor tissues and it is difficult to quantify only the mRNA within tumor cells. To solve this problem, the Danenberg tumor profile (DTP) which employs the microdissection technique was used. In this method, the tumor tissue is isolated from the excised prostatic sample for subsequent analysis, enabling a more accurate evaluation of the mRNA expression level.

If the gene expression of nucleic acid-metabolizing enzymes (represented by TS) is found to be a possible factor in determining prognosis, patients who are likely to exhibit a poor response would be separated from those undergoing the standard therapy in order to formulate the subsequent therapeutic approaches. Therefore, a retrospective study was conducted to discover whether the expression levels of TS, DPD, TP and OPRT (enzymes related to the metabolism of 5-FU drugs) can be used as prognostic markers.

\section{Patients and methods}

Case series. A total of 172 patients who were diagnosed with prostate cancer and underwent radical prostatectomies between 1996 and 2006 were recruited. Prostate tissues were collected from the surgical specimens and the relationship between the mRNA expression of nucleic acid-metabolizing enzymes and clinicopathological factors was retrospectively examined. Table I lists the backgrounds of the patients. Maximum androgen blockade was conducted in a neoadjuvant setting for 77 patients. The pathological specimens of the study were reviewed by a single pathologist. The median for the prostate-specific antigen (PSA) value at the initial examination was $7.2 \mathrm{ng} / \mathrm{ml}$ (range 2.8-111.2), while the median observation period was 4.05 years (range 0.6-16.3). Approval was obtained at commencement of the study from the ethics committee of our institution, and the determination and analysis of mRNAs were conducted on each of the 172 patients from whom informed consent was obtained.

Determination of the gene expression level of nucleic acidmetabolizing enzymes by RT-PCR. Following an examination of the hematoxylin and eosin (H\&E)-stained slides by the pathologist, the formalin-fixed, paraffin-embedded (FFPE) specimens were subjected to microtomy in order to prepare the slides. The mRNA (the level of gene expression) of each of the nucleic acid-metabolizing enzymes (TS, DPD, OPRT and $\mathrm{TP})$ contained in the tumor tissues on the slides was analyzed using the DTP method (Response Genetics, Inc., Los Angeles, CA, United States Patent No. 6,248,535). This method allowed the laser capture microdissection technique (11), by which tumor cells are selectively extracted from the FFPE, and the quantitative real-time RT-PCR method to be combined for relative quantitative determination of the mRNA of the target
Table I. Patient characteristics.

\begin{tabular}{lc} 
No. of patients & 172 \\
Median age (years) & 68 \\
Range & $49-77$ \\
Clinical stage & \\
B0 & 91 \\
B1 & 39 \\
B2 & 28 \\
C & 5 \\
Unknown & 9 \\
Histological type & \\
Well-differentiated & 65 \\
Moderately differentiated & 66 \\
Poorly differentiated & 17 \\
Gleason score & \\
6 & 17 \\
7 & 104 \\
8-10 & 41 \\
Median PSA (ng/ml) & 7.2 \\
Range & $2.8-111.2$ \\
\hline
\end{tabular}

gene. This combination was carried out to prepare the cDNA following RNA extraction, and a RT-PCR was conducted by the Taq Man method to amplify the target cDNA sequence. The expression level of each mRNA (for TS, DPD, TP or OPRT) was computed from the ratio against the expression of $\beta$-actin, a housekeeping gene.

Statistical analysis. The relationship between the expression level of mRNA for TS, DPD, TP or OPRT and the clinicopathological factors was evaluated: to analyze the differences in the mean of mRNA expression among the subgroups, $\log 10$ (mRNA) was used for a normal distribution; to compare two groups, a t-test was conducted in a state in which a parametric method was possible; and to compare three or more groups, tests were conducted by employing an analysis of variance. To examine whether the expression of each mRNA can be a prognostic factor, a receiver operating characteristic (ROC) analysis was conducted to obtain an appropriate cut-off value for each mRNA that was to be used for prognosis prediction. Additionally, the cases in this analysis were stratified according to the PSA relapse and the expression level of each mRNA (low or high): true positive (TP); false negative (FN); false positive (FP) and true negative (TN). Then, 'sensitivity $[=\mathrm{TP} /(\mathrm{TP}+\mathrm{FN})]$ ' and 'specificity $[=\mathrm{TN} /(\mathrm{FP}+\mathrm{TN})]$ ' were computed and the level of mRNA expression at which 'sensitivity - (1 - specificity)' is maximized was used as the cut-off value. For the analysis of survival time, PSA relapse-free survival (PSA-RFS) was computed by applying the KaplanMeier method and using the day of surgery as the starting point and the first day of the confirmed relapse of PSA as the day when the event occurred. To find the differences among subgroups, a log-rank test was used. The level of significance was set at $p<0.05$. Statistical software, JMP ver7.0.1 (SAS Institute, Inc.) was used for the statistical analysis. 
Table II. Relationship between clinicopathological factors and mRNA expression.

\begin{tabular}{|c|c|c|c|c|c|c|c|c|}
\hline & \multicolumn{8}{|c|}{ Mean of mRNA expression (n) } \\
\hline & $\mathrm{TS}$ & P-value & DPD & P-value & $\mathrm{TP}$ & P-value & OPRT & P-value \\
\hline \multicolumn{9}{|l|}{ Age (years) } \\
\hline$<65$ & $1.75(29)$ & \multirow[t]{2}{*}{ NS } & $0.81(24)$ & \multirow[t]{2}{*}{ NS } & $6.31(29)$ & \multirow[t]{2}{*}{ NS } & $0.94(28)$ & \multirow[t]{2}{*}{ NS } \\
\hline$\geq 65$ & $2.12(65)$ & & $0.82(59)$ & & $5.17(68)$ & & $0.96(64)$ & \\
\hline \multicolumn{9}{|l|}{ Stage } \\
\hline B0 & $1.83(59)$ & \multirow[t]{4}{*}{ NS } & $0.74(46)$ & \multirow[t]{4}{*}{ NS } & $4.22(52)$ & \multirow[t]{4}{*}{ NS } & $0.87(52)$ & \multirow[t]{4}{*}{ NS } \\
\hline B1 & $2.14(19)$ & & $0.98(14)$ & & $7.07(20)$ & & $1.06(19)$ & \\
\hline B2 & $1.94(12)$ & & $0.96(13)$ & & $5.22(14)$ & & $0.79(12)$ & \\
\hline $\mathrm{C}$ & 2.90 & & $0.87 \quad(4)$ & & 18.1 & & 1.53 (4) & \\
\hline \multicolumn{9}{|l|}{ Histological type } \\
\hline Well-differentiated & $1.99(39)$ & \multirow[t]{3}{*}{ NS } & $0.81(32)$ & \multirow[t]{3}{*}{ NS } & $5.67(40)$ & \multirow[t]{3}{*}{ NS } & $0.90(40)$ & \multirow[t]{3}{*}{ NS } \\
\hline Moderately differentiated & $1.80(40)$ & & $0.80(39)$ & & $5.64(42)$ & & $0.92(38)$ & \\
\hline Poorly differentiated & $2.32(9)$ & & $0.94 \quad(6)$ & & $4.51 \quad(9)$ & & 1.17 (9) & \\
\hline \multicolumn{9}{|c|}{ Including poorly differentiated type } \\
\hline- & $1.78(69)$ & \multirow[t]{2}{*}{$<0.05$} & $0.84(61)$ & \multirow[t]{2}{*}{ NS } & $5.93(72)$ & \multirow[t]{2}{*}{ NS } & $0.87(67)$ & \multirow[t]{2}{*}{$<0.05$} \\
\hline+ & $2.51(19)$ & & $0.73(16)$ & & 4.08 (19) & & $1.15(20)$ & \\
\hline \multicolumn{9}{|l|}{ Total Gleason score } \\
\hline 6 & 1.34 (6) & \multirow{3}{*}{ NS } & $0.68 \quad(5)$ & \multirow{3}{*}{ NS } & 3.29 (7) & \multirow{3}{*}{$<0.05$} & $0.70 \quad(6)$ & \multirow[t]{3}{*}{ NS } \\
\hline 7 & $1.97(68)$ & & $0.80(59)$ & & $4.74(69)$ & & $0.94(64)$ & \\
\hline $8-10$ & $2.34(19)$ & & $0.90(18)$ & & $8.53(20)$ & & $1.08(21)$ & \\
\hline \multicolumn{9}{|l|}{ PSA (ng/ml) } \\
\hline$<5.6$ & $1.80(30)$ & \multirow[t]{2}{*}{ NS } & $0.83(27)$ & \multirow[t]{2}{*}{ NS } & $4.70(33)$ & \multirow[t]{2}{*}{ NS } & $0.81(30)$ & $<0.05$ \\
\hline$\geq 5.6$ & $2.04(58)$ & & $0.82(51)$ & & $6.09(58)$ & & $0.99(57)$ & \\
\hline PSA relapse & & & & & & & & \\
\hline- & $1.81(68)$ & $<0.01$ & $0.87(62)$ & NS & $5.28(72)$ & NS & $0.86(68)$ & $<0.05$ \\
\hline+ & $2.55(20)$ & & $0.66(15)$ & & $4.06(19)$ & & $1.19(19)$ & \\
\hline Neoadjuvant therapy & & & & & & & & \\
\hline- & $2.00(74)$ & NS & $0.72(64)$ & $<0.01$ & $4.13(75)$ & $<0.01$ & $0.98(73)$ & $<0.01$ \\
\hline+ & $1.71(17)$ & & $1.21(17)$ & & $11.0(19)$ & & $0.65(16)$ & \\
\hline
\end{tabular}

TS, Thymidylate synthase; DPD, dihydropyrimidine dehydrogenase; TP, thymidine phosphorylase; OPRT, orotate phosphoribosyl transferase; NS, not significant.

\section{Results}

Evaluation of the level of enzyme expression (for TS, DPD, $T P$ and OPRT) related to 5-FU metabolism. The expression level for TS, DPD, TP and OPRT in the excised prostatic tissue was determined. The analysis was possible in 94, 83, 97 and 92 patients, respectively, and the mean level of expression for each mRNA was $1.805,0.69,3.52$ and 0.85 , respectively.

A comparative evaluation was performed in order to investigate possible correlations between the mRNA expression of each enzyme (TS, DPD, TP or OPRT) and various clinicopathological factors related to prostate cancers. Table II shows the mean of mRNA expression. No significant differences were found in patient age, tumor stage and differentiation or level of mRNA expression. However, the expression of TS and OPRT was significantly higher in those cases exhibiting poorly differentiated carcinoma in their tumor tissues. When contrasted to the total Gleason scores, the increase from 6 to 7 , then to $8-10$ was positively correlated with increases in
TS, DPD, TP and OPRT expression; however, only TP showed a significant difference. Regarding the correlation between the levels of PSA at the initial examination and the mRNA expression, the OPRT expression was significantly higher in the 'PSA-high' group ( $\geq 5.6 \mathrm{ng} / \mathrm{ml}$ ) in contrast to the 'PSA-low' group $(<5.6 \mathrm{ng} / \mathrm{ml})$. Among the patients in whom PSA relapse was confirmed, the expression of TS and OPRT was significantly higher. When the groups with and without pre-surgical treatment were compared, the expression of DPD and TP was significantly higher and that of OPRT significantly lower in the former group.

The expression of TS, DPD, TP and OPRT and its potential role as a prognostic factor. To evaluate the potential use of each mRNA expression as a prognostic factor, the PSA-RFS was examined for the expression of each mRNA. The cut-off values for mRNAs for TS, DPD, TP and OPRT determined by the ROC analysis were 2.98, 0.84, 3.09 and 0.68, respectively. mRNAs exceeding the cut-off values were defined as the high 


\section{TS mRNA}

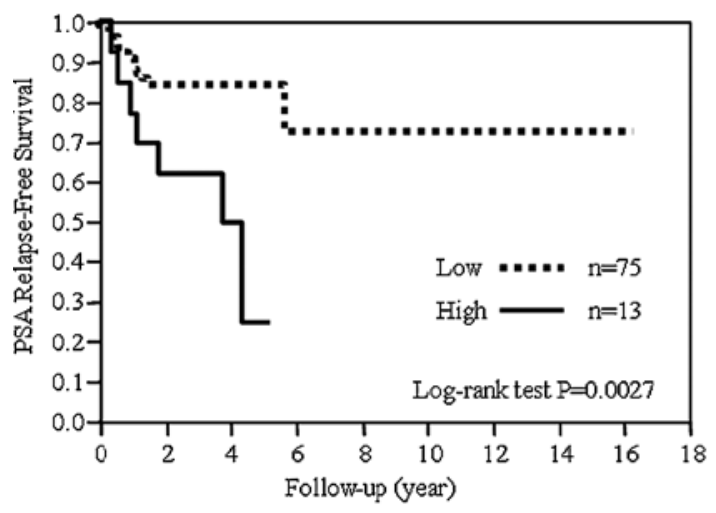

TP mRNA

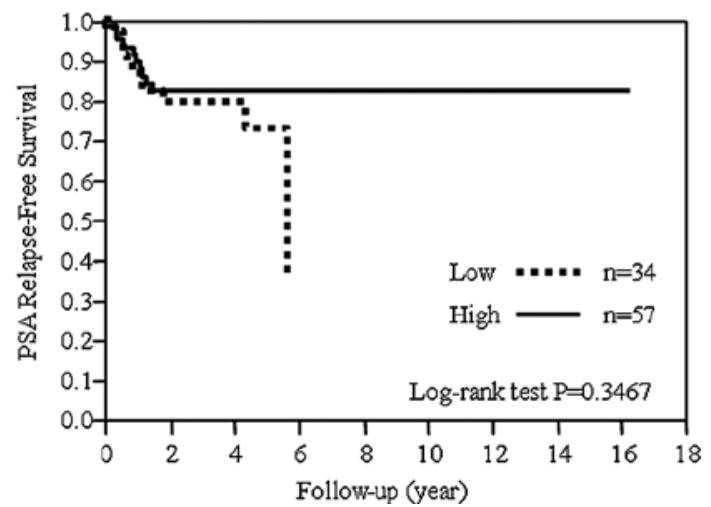

DPD mRNA

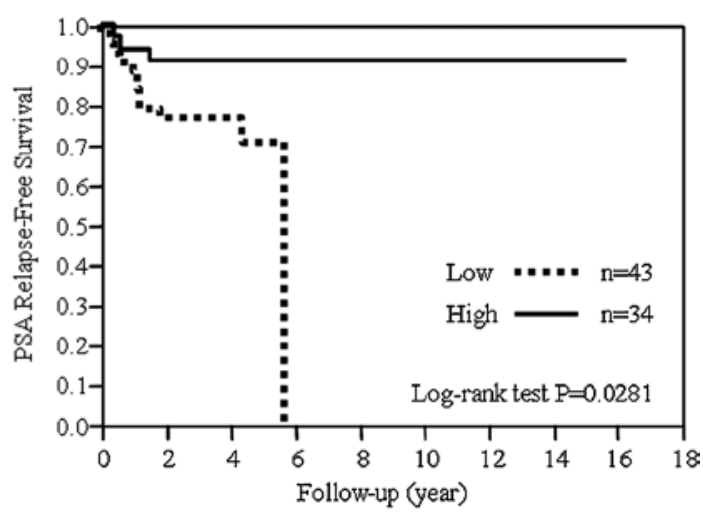

OPRT mRNA

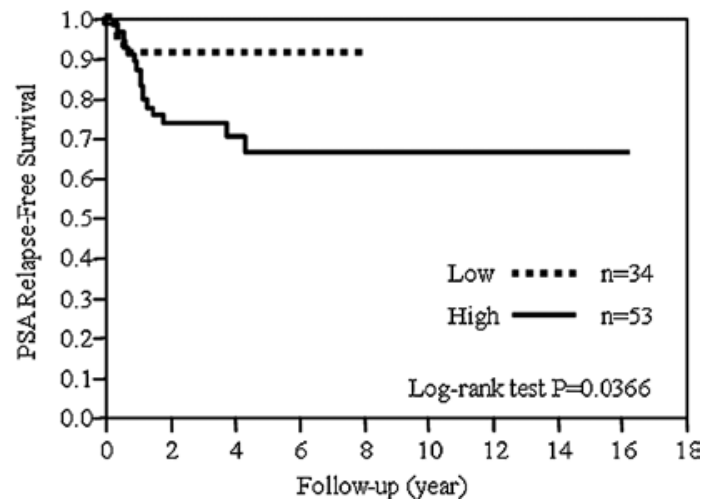

Figure 1. PSA relapse-free survival according to mRNA expression level. Relationship between PSA relapse-free survival and mRNA expression of TS, DPD, TP and OPRT. mRNA were divided into two groups with a high and low expression.

groups and those below the cut-off values, the low groups, and their correlation with prognosis was examined. The prognosis was significantly poorer in the TS-high, DPD-low and OPRThigh groups (Fig. 1).

The 5-year PSA-RFS was 84, 24.62, 70.78, 91.08, 72.59, $82.25,91.18$ and $66.2 \%$ in TS-low and -high, DPD-low and -high, TP-low and -high and OPRT-low and -high groups, respectively.

\section{Discussion}

In the present study, the results of the analysis of relapse-free survival time stratified by the mRNA expression indicated that the prognosis is significantly poorer in cases when the TS mRNA level was over 2.98 , OPRT mRNA exceeded 0.68 or the DPD expression level was below 0.84. It was suggested that these cases require additional treatment or that the planning of a new therapeutic modality was necessary to improve prognosis. Studies on the relationship between mRNA expression and prognosis have been conducted on various types of tumor, but only a few focused on prostate cancers (10). The data presented in this study are beneficial to future studies.

TS is known to be a target enzyme for 5-FU, and its potential role as a prognostic factor has been described for various types of tumor species. Patients with positive or high levels of TS exhibited a poor prognosis (12-15), which was in agreement with our results. DPD is a 5-FU decomposing enzyme and is crucial for determining sensitivity to this anticancer agent. Previous studies found that cases that were exposed to 5-FU preparations and showed a high DPD level responded poorly to these therapeutic agents or the prognosis was found to be poor (16-18). These cases had been treated with 5-FU preparations and it was considered that the DPD expression levels affected the drug effects. In this study, few patients were exposed to 5-FU preparations, but contrary to the results of the earlier studies, the prognosis was poor in the DPD-low group. TP did not affect prognosis in the present study, but it was reported that in cases of metastatic colorectal cancer the effect of 5-FU is limited when the TP mRNA expression is high in the tumor tissue (19). In another study, a satisfactory prognosis is described in those cases with high levels of TP expression among patients who underwent post-operative adjuvant chemotherapy (20). OPRT is a 5-FU phosphorylating enzyme. In cancers of the digestive system, OPRT defines the prognosis and 5-FU sensitivity, and patients with a low expression of this enzyme are associated with a poor prognosis $(13,21,22)$. Results of this study, however, showed that a poor prognosis in the group with a high OPRT expression. Of note is that the relationship between each mRNA expression and prognosis varies, depending on the type of cancer.

Compared to tumors affecting other organs, prostate cancers are characterized by tumors scattered throughout the prostate gland. Therefore, macroscopic differentiation of the tumor from normal tissues is difficult. In early tumors 
with a surgical indication, in particular, the tumor volume is limited, making the diagnosis even more difficult. In addition, tumor and interstitial tissue are intermingled. Subsequently, when mRNA is extracted directly from the tumor tissue of an excised specimen, the expression level of mRNA in the tumor cells may not be evaluated accurately. In the present study, on the other hand, the DTP method was utilized and it was hypothesized that the volume of the mRNA expressed was accurately determined. However, the DTP method also has limitations, such as non-availability under certain conditions for the preservation or fixation of the FFPE specimens and limitations regarding facilities where the determination can be made. When compared to immunostaining, the DTP method is more beneficial due to accurate quantification of mRNA expression. It is crucial to select a method of determination that is most suitable for the research objective.

In examining the mRNA expression of these enzymes in cancerous prostatic tissue, the group with high PSA levels at the initial examination exhibited significantly higher levels of OPRT mRNA expression, indicating the possibility that OPRT is a prognostic factor for prostate cancers. In the cases exhibiting a high expression of TS and OPRT mRNA, including certain cases with poorly differentiated carcinoma, this suggests that a correlation with malignant potential exists.

When a group of patients who had received pre-operative treatment was compared to patients who had not such treatment, it was noted that in the former group, the DPD and TP mRNA expression was high, whereas the OPRT mRNA expression was low. Few studies have reported that the pre-operative endocrine therapy of prostate cancers affects the level of mRNA expression of nucleic acid-metabolizing enzymes (23). It is possible that the pre-operative treatment exerted a direct effect on the mRNA expression patterns in the tumor tissue, or a relative decrease of tumor cells that are considered to be associated with high malignancy resulted in an apparent change in the expression level. However, the mechanism by which endocrine therapy affects nucleic acid-metabolizing enzymes is inadequately elucidated in this study.

This study was conducted on patients with localized cancers, but the determination of mRNA of these enzymes may also contribute to the prognostic evaluation of patients with advanced cancers. If individualized treatment becomes a reality by using prognostic factors, selective treatment may become an option for patients in whom 5-FU preparations show more promising results.

In conclusion, among patients with prostate cancers, those with a poor prognosis can be identified by determining the levels of TS, DPD and OPRT mRNA expression in the excised tumors. Such an mRNA expression appears to be useful as a prognostic factor.

\section{References}

1. Mok TS, Wu YL, Thongprasert S, et al: Gefitinib or carboplatinpaclitaxel in pulmonary adenocarcinoma. N Engl J Med 361: 947-957, 2009.

2. Heggie GD, Sommadossi JP, Cross DS, Huster WJ and Diasio RB: Clinical pharmacokinetics of 5-fluorouracil and its metabolites in plasma, urine, and bile. Cancer Res 47: 2203-2206, 1987.
3. Van Kuilenburg AB: Dihydropyrimidine dehydrogenase and the efficacy and toxicity of 5-fluorouracil. Eur J Cancer 40: 939-950, 2004.

4. Danenberg PV: Thymidylate synthetase - a target enzyme in cancer chemotherapy. Biochim Biophys Acta 473: 73-92, 1977.

5. Pinedo HM and Peters GF: Fluorouracil: biochemistry and pharmacology. J Clin Oncol 6: 1653-1664, 1988.

6. Longley DB, Harkin DP and Johnston PG: 5-Fluorouracil: mechanisms of action and clinical strategies. Nat Rev Cancer 3: 330-338, 2003.

7. Peters GJ, Laurensse E, Leyva A, Lankelma J and Pinedo HM: Sensitivity of human, murine, and rat cells to 5-fluorouracil and 5'-deoxy-5-fluorouridine in relation to drug-metabolizing enzymes. Cancer Res 46: 20-28, 1986.

8. Peters GJ, van Groeningen CJ, Laurensse EJ and Pinedo HM: A comparison of 5-fluorouracil metabolism in human colorectal cancer and colon mucosa. Cancer 68: 1903-1909, 1991.

9. Fukui Y, Oka T, Nagayama S, Danenberg PV, Danenberg KD and Fukushima M: Thymidylate synthase, dihydropyrimidine dehydrogenase, orotate phosphoribosyltransferase mRNA and protein expression levels in solid tumors in large scale population analysis. Int J Mol Med 22: 709-716, 2008.

10. Li Y, Mizutani Y, Shiraishi T, et al: Prognostic significance of thymidylate synthase expression in patients with prostate cancer undergoing radical prostatectomy. Urology 69: 988-995, 2007.

11. Emmert-Buck MR, Bonner RF, Smith PD, et al: Laser capture microdissection. Science 274: 998-1001, 1996.

12. Ichikawa W, Takahashi T, Suto K, et al: Thymidylate synthase predictive power is overcome by irinotecan combination therapy with S-1 for gastric cancer. Br J Cancer 91: 1245-1250, 2004.

13. Ichikawa W, Takahashi T, Suto K, et al: Simple combinations of 5-FU pathway genes predict the outcome of metastatic gastric cancer patients treated by S-1. Int J Cancer 119: 1927-1933, 2006.

14. Hakamada Y, Tsuchida A, Arima M, et al: Prognostic predictors in breast cancer patients with postoperative 5-fluorouracil-based chemotherapy. Int J Mol Med 16: 309-314, 2005.

15. Miyoshi T, Kondo K, Toba H, et al: Predictive value of thymidylate synthase and dihydropyrimidine dehydrogenase expression in tumor tissue, regarding the efficacy of postoperatively administered UFT (tegafur+uracil) in patients with non-small cell lung cancer. Anticancer Res 27: 2641-2648, 2007.

16. Etienne MC, Chéradame S, Fischel JL, et al: Response to fluorouracil therapy in cancer patients: the role of tumoral dihydropyrimidine dehydrogenase activity. J Clin Oncol 13: 1663-1670, 1995.

17. Horiguchi J, Yoshida T, Koibuchi Y, et al: DPD activity and immunohistochemical DPD expression in human breast cancer. Oncol Rep 11: 65-72, 2004.

18. Salonga D, Danenberg KD, Johnson M, et al: Colorectal tumors responding to 5-fluorouracil have low gene expression levels of dihydropyrimidine dehydrogenase, thymidylate synthase, and thymidine phosphorylase. Clin Cancer Res 6: 1322-1327, 2000.

19. Metzger R, Danenberg K, Leichman CG, et al: High basal level gene expression of thymidine phosphorylase (platelet-derived endothelial cell growth factor) in colorectal tumors is associated with nonresponse to 5-fluorouracil. Clin Cancer Res 4: 2371-2376, 1998

20. Saito S, Tsuno N, Nagawa H, et al: Expression of plateletderived endothelial cell growth factor correlates with good prognosis in patients with colorectal carcinoma. Cancer 88: 42-49, 2000.

21. Ochiai T, Nishimura K, Noguchi $\mathrm{H}$, et al: Prognostic impact of orotate phosphoribosyl transferase among 5-fluorouracil metabolic enzymes in resectable colorectal cancers treated by oral 5-fluorouracil-based adjuvant chemotherapy. Int J Cancer 118: 3084-3088, 2006

22. Ichikawa $\mathrm{W}$, Uetake $\mathrm{H}$, Shirota $\mathrm{Y}$, et al: Both gene expression for orotate phosphoribosyltransferase and its ratio to dihydropyrimidine dehydrogenase influence outcome following fluoropyrimidine-based chemotherapy for metastatic colorectal cancer. Br J Cancer 89: 1486-1492, 2003.

23. Inoue T, Segawa T, Shiraishi T, et al: High-grade and hormonetreated prostate cancer express high levels of thymidylate synthase. BJU Int 98: 197-200, 2006. 